\section{SUBJECT INDEX \\ VOLUME 45, 2003}

Abadiyeh/Hu, 123-130

Accelerator Mass Spectrometry (AMS), 1-7, 81-89, 421-430, 431-447, 449-466

Age-depth relationship, 501-506

Age shifts, 9-15

Air-sea exchange of $\mathrm{CO}_{2}, 431-447$

Amino acids, 409-419

Arabian Sea, 467-477

Archaeology, 59-73, 101-112

Atmospheric transport, 431-447

Automated system, 421-430

Balanced window method, 113-122

Bioturbation, 501-506

Bones, 409-419

Bronze Age, 41-58

Burial mounds, 101-112

Cantabrian Spain, 41-58

Castor oil contamination, 497-499

Central Argentinian Andes, 33-39

Central equatorial Pacific, 91-99

Chalcolithic, 41-58

Chronology, 59-73

Coral radiocarbon, 91-99

Dead Sea Scrolls, 497-499

Dendrochronology, 431-447

Denmark, 101-112, 449-466

Depth error, 501-506

Early human peopling, 33-39

Egypt, 123-130

Europe, 449-466

Fatty acids, $17-24$

Floating varve scale, 467-477

Food residue, 449-466

Fourth International Radiocarbon Intercomparison (FIRI), 75-80, 135-408, 493-495

freshwater fish, 449-466

GISP-2 correlation, 467-477

Gruta del Indio, 33-39

Holocene climatic changes, 25-32

Index of humidity, 25-32

Ink rubbing, 1-7
Last Glacial Maximum (LGM), 467-477

Liquid scintillation counting (LSC), 113-122

Mesolithic, 41-58

Methods, 421-430

Nagada, 123-130

Neolithic, 41-58

New Zealand, 479-491

Ninhydrin, 409-419

Organic samples, 421-430

Pacific Decadal Oscillation, 91-99

Paleolithic (Upper and Middle), 41-58

Patagonia, 9-15

Peat sort, 25-32

Pleistocene megafauna, 33-39

Pollen, 25-32

Pollen dating, 479-491

Pottery, 449-466

Predynastic, 123-130

Quaternary, 479-491

Radiocarbon, 9-15, 431-447

Radiocarbon age, 467-477

Radiocarbon calibration, 81-89

Radiocarbon dating, 17-24, 25-32, 41-58, 101-112, 409-419, 449-466

Regional calibration, 81-89

Reservoir effect, 9-15, 449-466

Ross Sea, 17-24

Sample preparation, 1-7, 479-491

Scanning electron microscope (SEM), 1-7

Semaineh, 123-130

Soil organic matter, 101-112

South America, 59-73

Statistical analysis, 59-73

Third International Radiocarbon Intercomparison (TIRI), 75-80, 135-408

Treatment, 409-419

Tree rings, $431-447$

Turbidite, $75-80$

West Antarctic Ice Sheet (WAIS), 17-24

Wiggle-matching, 81-89 